\title{
Streptomycin Sulfate
}

National Cancer Institute

\section{Source}

National Cancer Institute. Streptomycin Sulfate. NCI Thesaurus. Code C47729.

The sulfate salt form of streptomycin, an aminoglycoside antibiotic derived from

Streptomyces griseus with antibacterial property. Streptomycin sulfate binds to the S12 protein of the bacterial 30 S ribosomal subunit, thereby inhibiting peptide elongation and protein synthesis, consequently leading to bacterial cell death. 